\title{
Dynamics of subjective change in psychotherapy. A Comment on the Paper "Dynamics of sense-making and development of the narrative in the clinical exchange" by Alessandro Gennaro, Miguel Gonçalves, Inês Mendes, António Ribeiro, \& Sergio Salvatore
}

Mariane Krause $e^{1} \&$ Claudio Martínez ${ }^{2}$

The following comment has been divided into four sections that present a critical discussion on: (1) the theoretical background of the paper; (2) the two methods used: Discourse Flow Analysis (DFA) and Innovative Moments Coding System (IMCS); (3) results; and (4) conclusions.

\section{Theoretical background}

The article is based on a widely accepted notion of psychotherapeutic change, which considers that change takes place in the representational sphere (Fonagy, 2001). This notion of change is derived from the Theory of Subjective Change (Krause, 2005) and has been referenced through concepts such as: changes in the frames of reference (Duncan \& Moynihan, 1994), changes in personal constructs (Anderson, 1997a, 1997b), or change as a "re-writing" of aspects of one's life story (McLeod, 1998; McLeod \& Balamoutsou, 1996).

\footnotetext{
${ }^{1}$ Escuela de Psicología, Pontificia Universidad Católica de Chile.

${ }^{2}$ Facultad de Psicología, Universidad Diego Portales.

E-mail: mkrause@uc.cl
}

(Received 9 June 2011; Revised 21 June 2011; Accepted 8 July 2011) 
The authors allude to this idea of change through the concept of "sense making." In fact, they define "psychotherapy as an intersubjective dynamics of sense-making aimed at changing a patient's symbolic (affective and/or cognitive) modality of interpreting his/her experience" (Gennaro, Gonçalves, Mendes, Ribeiro, \& Salvatore, 2011, p. 91). This definition fully matches that of subjective change (Krause, 2005), which regards a change in meanings as the core of psychotherapeutic change. The concept of "sense making" is closely associated with the hermeneutic or interpretive epistemological tradition, and leads to the use of methodologies capable of unlocking the meanings that therapeutic communication has for its participants.

When they focus on the process, the authors articulate the interpretive perspective with a constructionist one, characterizing psychotherapy as " 'transformative dialog' (Gergen, 1999, p. 250), where new meanings are elaborated, new categories are developed, and one's presuppositions (Chambers \& Bickhard, 2007) are transformed within and thanks to the interpersonal context" (Gennaro et al., 2011, p. 91). In this regard, two notions highlighted by the authors are noteworthy. One of them is Gergen's notion of "transformative dialog" -quoted by the authors- which implies the idea of dialogic transformation. This idea seems to be coherent with the notion of flow in the DFA system and with the implicit idea of two minds constructing meaning together, that is, in a dia-log. In other words, the DFA system seems to incorporate a concept of change which highlights the possibility that two minds transform when sharing a novel experience in a dynamic space. The second remarkable idea, which complements the former, is that of "semiotic novelty," since it stresses the power of the coconstructive in the form of construction of the dialog, rather than in its meanings. Novelty may be said to act as a lever of change for the patient; it is the different way in which something is told or narrated, and new meanings are thought to emerge from such narrative novelty. This can also be understood based on the notion of alterity (Bakhtin, 1986), which highlights the richness of strange voices in the way of telling something. The 
other (alter) provides the difference, which becomes novelty, just for being somebody else and thinking differently. Therefore, this experience of novelty with an other sets the stage for the transformation of an individual's own experience. The transformation can be expressed, for example, in the new way that a patient finds to narrate his/her experience. This is coherently illuminated by the IMCS system, which values the expression of change based on the narrative novelty expressed by the patient, which is heard (read) by an observer-researcher, who then transforms it into a new narration in the form of ordered categories. The key part of these processes appears to be the presence of an other (alter) who, as a dialogic actor, sanctions this novelty and incorporates it, making it his/her own in the form of psychological change.

Consistent with this manner of focusing on the process, the authors believe that "sense-making could be depicted as a dynamic process, that is a process depending on time" (Gennaro et al., 2011, p. 91). The idea of the evolution of change over time is also supported by theoretical and empirical information, especially concerning the progression of stages, for example, in Stiles' model of assimilation of problematic experiences (2002), in Hill's Three-stage Model of Helping (2001), in the Generic Change Indicators developed by Krause et al. (2007), or in the transtheoretical model of Prochaska and Norcross (2002).

Fortunately, given the proliferation of methods to assess change during the process (and in terms of outcome), psychotherapy research has reached a point of conceptual consensus about the essence of the product of psychotherapeutic change and of its construction process. This consensus makes it possible to advance in the development of a generic and inclusive theoretical model, which is capable of articulating specific studies and giving coherence to its multiplicity. This also fosters the connection between research and clinical practice, since a clinical professional will prefer a study that delivers more comprehensive results instead of being swamped with hundreds of specific and microscopic studies. 


\section{Methods}

The article presents two methods: the Discourse Flow Analysis (DFA) and the Innovative Moments Coding System (IMCS). The DFA searches for the "dynamics of sense-making", looking at connections of meanings. IMCS, in contrast, seeks unique and novel content in therapeutic discourse, in the form of narrations which emerge over the course of the therapeutic process. Through the application of both methods to a case, the authors intend to establish "the relationship between the formal and functional mapping of a psychotherapy case and the content of the patient's narrative" (Gennaro et al., 2011, p. 94). "The main aim is to chart out which kind of movements at level of narrative content corresponds to the dynamics of sensemaking carried out by the clinical dialogue as depicted by the DFA" (Gennaro et al., 2011, p. 94).

In theoretical terms, DFA involves two phases in the evolution of the change of meanings during the therapeutic process: a deconstructive stage followed by a constructive one. Dysfunctional meanings are deconstructed so that new meanings can emerge afterwards. This means that, at the beginning of the therapy, meanings should be more rigid; then, they should become unfrozen, and, finally, a new consolidation should take place, made up by meanings constructed in the therapy. A parallel can be drawn between this notion and the evolution of Generic Change Indicators, which also involve an early "melting" moment followed by the consolidation of new meanings in later phases (Krause et al., 2007). Therefore, the rigidity of meanings is thought to evolve over the therapy in a U-shaped fashion.

Methodologically, "DFA assumes that sense-making depends on the associations for temporal adjacency between meanings [...]. Accordingly, DFA maps the psychotherapeutic dialogue in terms of associations for adjacency between semantic contents (i.e. the fact that one meaning comes just after another) occurring within the clinical exchange" (Gennaro et al., 2011, p. 97).

DFA works with (previously segmented) textual transcriptions which then undergo computer-aided content analysis. The second step of DFA is 
sequential: "This procedure calculates each semantic content's probability of coming straight after every thematic content" (op. cit., p. 99). The third step is the establishment of super-ordered nodes, based on their frequency of occurrence and their association with other nodes. The presence of such super-ordered nodes is what results in the "U-shape trend" visible over the course of the psychotherapy.

The heart of the DFA method seems to be in its third step, that is to say, the analysis of the formal characteristics of the discursive network through the identification of the main nodes (semiotic set or semiotic entity), followed by the quantification of the connections between these two nodes, their distribution, and their connective trajectories. In this analysis, the key element appears to be the Super-Ordered Nodes (SN), which group connections according to their frequency and type. This is interesting because it can be a way of describing regulatory and self-regulatory instances in discourse. Depending on the trajectory or direction of the connections, it may be possible to identify predominantly self-regulatory nodes and nodes that tend to participate as regulators of interaction with the other. Also, it might be feasible to identify more or less active regulatory patterns depending on the stage of the therapeutic process. This would make it possible to observe changes through the flow of connections and disconnections of these patterns during the process, and to establish the direction of such changes along with the influence of interaction in all of these movements.

In terms of its coherence with the epistemological background discussed in the first section of this commentary, DFA does not deal with the meanings of the patient's discourse or narrative in depth; instead, it supposes that a given frequency of signs or repeated trajectories of connections of signs (words, utterances) result in the meaning of a given sign or set of signs. In this regard, the method deviates from the hermeneutic tradition.

The second method -The Innovative Moments Coding System (IMCS)- is introduced in the article as a qualitative procedure which analyzes the contents of the patient's narrative. This analysis is conducted using 
Innovative Moments (Gonçalves, Matos, \& Santos, 2008, 2009), which, based on a conception of psychotherapy as narrative, refer to a discursive content which emerges as a novelty in the story that the patient usually narrates about his/her problem (White \& Epston, 1990). Consequently, IMs reflect a clinical change from their expression in the patient's narrative, and are therefore consistent with a semiotic conception of the psychotherapeutic process and with the idea of novelty as a precursor of therapeutic change.

Both conceptions are shared by the DFA method, but where this method seems to define a "skeleton" in the form of a discursive network, the IMCS adds the "flesh" of content. Furthermore, the two methods conceive novelty in epistemologically different ways. While in DFA semiotic novelty emerges through the connection of symbols which acquire meaning as they form spatially significant groups, novelty in the IMCS method is sanctioned by a third party (researcher) who uses pre-established representational categories to do so.

In terms of their assumptions (which are used as hypotheses in this study) about the evolution of the psychotherapeutic process, both methods are comparable, given that the U-shape in the evolution of the superordered nodes proposed by DFA is compatible with the type of I-Moments suggested by IMCS. According to the latter, out of the five types of IMoments (Action, Reflection, Protest, Re-conceptualization, Performing Change), the first three can be grouped under a category "that represents a rupture in respect to the dominant narrative" (Gennaro et al., 2011, p. 106), while the last two can be seen as being part "of an elaborative process producing a consolidation of the new perspectives" (op. cit., p. 106). Thus, both methods propose two general phases in the "ideal" course of good outcome psychotherapies. The study, then, seeks to link "reactive innovation" with the deconstructive phase of DFA, and "elaborative" innovation with its "constructive" equivalent-this is the central hypothesis about the complementation of the two methods.

One of the virtues that these methods share is their appropriateness for the sequential analysis of the psychotherapeutic process. According to the 
authors, the methods not only reveal what is said, and how it is said, but also when what said is said (what comes before and after what is said). This allows us to achieve a profound and dynamic understanding of psychotherapeutic change.

Regarding their differences, the authors point out that IMCS, in contrast to DFA, focuses on the content level. However, to a certain extent, both methods deal with content, not only IMCS. In fact, the starting point of DFA is the definition of topics (content) and their frequencies, followed by the establishment of cross-sectional connections (in a segment) along with longitudinal ones (between segments, over time). The difference between them is that, in DFA, contents are used without an a priori "sense-making" point, as is the case of IMCS, and become sets of signs grouped by frequency or lemmas. Thus, in DFA, initial contents become formal structures and flows. In contrast, IMCS stays mostly within the dimension of contents which become relevant when a coder grants a "novel" place in discourse to a certain narrative. The transit towards formality only occurs in the classification stage of types of I-Moments and then in the determination of the salience of IMs within a session or group of sessions. This transit towards the formal dimension allows the combination of methods and supports the study hypothesis.

\section{Results}

The study hypotheses were: In the first stage of therapy (sessions 1 to 10): (a) "a negative association between the SN [Super-Ordered Nodes] trend and the duration of the [...] reactive i-moments" (Gennaro et al., 2011, p. 108); (b) no association "between the SN's trend and the elaborative imoments" (op. cit., p. 108). In the second stage of therapy (sessions 11 to 15); (c) a positive association between the SN and the elaborative IMs [Innovative Moments]; (d) the same negative association between the SN and the reactive i-moments.

Firstly, it is important to note that the results generated with the DFA support the U-shape in the evolution of Super-Ordered-Nodes (SN) in this 
successful therapy. In this context, the aforementioned hypothesis could be tested. The results obtained with IMCS reveal that reactive IMs (their duration) show an inverse U-shape, while elaborative IMs increase only in the second stage of therapy. Furthermore, SNs have a negative correlation with reactive IMs during both stages of therapy, and a positive correlation with elaborative IMs only in the second stage. These results support the main hypothesis of the study. In other words, they show that both methods are capable of "drawing" the trajectory of this therapy, and are consistent with respect to each other.

In conceptual terms, the authors conclude that the "weakening of the initial patient's assumptions have created the room for the emergence of innovative meanings challenging the dominant narrative [...]. After and thanks to this first phase, that has lasted two thirds of the therapy, Lisa has had the opportunities to elaborate new super-ordered meanings" (op. cit., pp. 113-114). This causal interpretation may be disputable, especially considering that it is a single case. Another debatable point is the implicit assumption that, if something is deconstructed, something better will be constructed in its place. At this point, it is worth focusing on the lower portion of the U-shape, since what is constructed afterwards depends on what has happened there.

\section{Conclusions}

The results are relevant in many ways: a) they have methodological value, as they "cross validate" two different analysis techniques; b) they show the evolution of the therapeutic process; c) they provide an in-depth analysis of the changes of meaning in therapeutic dialog. Regarding their contribution to the understanding of therapeutic change, in terms of the notion of motion and time, especially DFA makes it possible to "draw" this temporality, mapping the paths that crisscross it. The contribution of the system lies in mapping such flows and movements. What is complex is to make sense of these flows, of this transit. This is attempted through IMCS in 
the article, with the objective of adding "narrative meat" to the forms and functioning of the dialog.

In spite of these undeniable contributions, two aspects deserve a more critical discussion, since they allow for points of view different from those of the authors.

The first one, already mentioned in our comment about the study results, refers to how dynamics are understood in comparison with contents, and how both dimensions are connected. According to the authors, "findings highlight the association between the formal and functional characteristics of the clinical dialogue and the content of the narrative" (op. cit., p. 90). The problem is that, if we consider the methodological procedure of DFA in detail, it becomes clear that the method is based on the contents of therapeutic discourse. Therefore, both methods are -to some extent- based on the contents of clinical dialog. Why the association between contents -which is the specific contribution of DFAshould be conceptualized as "formal and functional characteristics of the clinical dialogue" or (only) as dynamics is not self-evident, although the reason may be that, in DFA, contents are not used as representations but as signs. Semantic contents are only relevant for DFA as they warrant the use of a computer mechanism to group them, but this grouping loses all its referential meaning due to the deconstruction of the patient's discourse that the method entails.

Regarding the relationship between dynamics and content, the authors hold that their results describe the dynamics of sense-making sustaining the psychotherapy process provided by DFA and the contents of the narrative that such dynamics produce. Can dynamics and content be separated? (Especially considering that the method that focuses on dynamics is supported by contents); is not this tantamount to separating dancing from the dancer? (Orlinsky, 2007). To pursue the metaphor: the dynamics of therapeutic dialog do not "cause" contents, just as dancing does not "cause" the dancer. The separation of the two dimensions has mainly methodological aims, and is the most artificial part of the method. 
Finally, the authors explicitly intend for their results to have clinical relevance. The question is whether the I-Moments methodology is enough to establish this clinical relevance. In other words, the authors assume that Innovative Moments are equivalent to "clinical value," and therefore, that the connection between the methods can be regarded as a "clinical validation" of sorts that IMs grant to DFA. Needless to say, both methods (like all methods) have a certain degree of artificialness, and thus lack an immediate connection with the clinical dimension; however -and we agree with the authors concerning this point- IMs are qualitatively closer than DFA to the meanings that the actors intend to transmit through their discourse.

On the other hand, regardless of the abstractness of DFA results or IMoments categorization, they can have clinical relevance because they represent therapy evolution models. So, it can be relevant for every psychotherapist to know that the first phase of the therapy must accomplish the permeability of super-ordered meanings, to then assist the patient in constructing new meanings which are more functional to his/her psychological wellbeing.

In contrast, a researcher will find it interesting to discover the formal structure of psychotherapeutic change trajectories, and check whether it repeats itself across different therapy types, or if it changes depending on the success of the therapy. The contribution of the methods in this regard is to provide evidence that the dynamics and flow of dialog are not random or spontaneous, but that they are a construction of meaning which is shaped over time. Although these paths may seem emergent and chaotic, they can attain a novelty of meaning through analysis. Some questions that the researchers can explore in the future: Despite novelty and emergence, are there any paths that repeat themselves in all psychotherapies? Are there any similar trajectories beyond individual novelty? Does the therapeutic process follow similar lines over time? Ideally, the results that emerge from these studies will also nourish clinical practice, if they manage to be translated into a clinically meaningful language. 
Thus, we must ask ourselves about the level (in the theoretical-practical dimension) to which these results should be taken for them to be truly useful for clinicians. A new step in this direction could be to link the trajectories of change with therapeutic work, so that clinicians may also obtain information about how the initial phase is "deconstructed" and how the final one is "constructed." For example: Is the interpretation of resistance at the beginning of the therapy useful as a way to force the "deconstruction" of old patterns? Or, are the therapist's actions unimportant because the initial Super-Ordered Nodes are fed by the patient him/herself?

\section{References}

Anderson, R. H. (1997a). Conversation, Language, and Possibilities: A Postmodern Approach to Therapy. New York: Basic Books.

Anderson, R. H. (1997b). Interpreting therapeutic process: A constructivist perspective. Journal of Constructivist Psychology, 10, 297-319.

Bakhin, M. (1986). Speech Genres and Other Late Essays. Austin: University of Texas Press.

Duncan, B. L., \& Moynihan, D. W. (1994). Applying outcome research: intentional utilization of the client's frame of reference. Psychotherapy, 31, 294-301.

Fonagy, P. (2001). El proceso del cambio y el cambio de los procesos: qué puede cambiar en un "buen análisis" [The Process of Change and the Change of the Processes: What Can Change in a "Good Analysis"]. Revista Chilena de Psicoanálisis, 18, 80-96.

Gergen, K. J. (1999). An invitation to Social Construction. London: Sage.

Gonçalves, M. M., Matos, M., \& Santos, A. (2008). Innovative Moments Coding System. Version 7.0. Braga, Portugal: University of Minho. (Available from the authors).

Gonçalves, M. M., Matos, M., \& Santos, A. (2009). Narrative therapy and the nature of "innovative moments" in the construction of change. Journal of Constructivist Psychology, 22, 1-23. 
Hill, C. (2001). Helping Skills: The Empirical Foundation. Washington, DC: American Psychological Association.

Krause, M. (2005). Psicoterapia y Cambio [Psychotherapy and Change]. Santiago, Chile: Ediciones Universidad Católica.

Krause, M., De la Parra, G., Arístegui, R., Dagnino, P., Tomicic, A., . . . Ramírez, I. (2007). The evolution of therapeutic change studied through generic change indicators. Psychotherapy Research, 17, 673-689.

McLeod, J. (1998). Narrative and Psychotherapy. London: Sage.

McLeod, J., \& Balamoutsou, S. (1996). Representing narrative process in therapy: Qualitative analysis of a single Case. Counselling Psychology Quarterly, 9, 61-76.

Orlinsky, D. E. (2007). ¿Qué me Gustaría Leer Durante los Próximos 10 años de Investigación en Psicoterapia? [What Kind of Psychotherapy Research Would I Like to Read During the Next 10 Years?]. Invited address in the VII Congreso Latinoamericano de Psicoterapia y I Congreso Uruguayo de Psicoterapia, Federación Uruguaya de Psicoterapia. Montevideo (Uruguay).

Prochaska, J. O., \& Norcross, J. C. (2002). Stages of Change. In J. C. Norcross (Ed.), Psychotherapy Relationships that Work: Therapist Contributions and Responsiveness to Patients (pp. 303-313). New York: Oxford University Press.

Stiles, W. B. (2002). Assimilation of Problematic Experiences. In J. C. Norcross (Ed.), Psychotherapy Relationships that Work: Therapist Contributions and Responsiveness to Patients (pp. 357-365). New York: Oxford University Press.

White, M., \& Epston, D. (1990). Narrative Means to Therapeutic Ends. New York: Norton. 